Received: 11 August 2017

Accepted: 14 February 2018

Published online: 22 February 2018

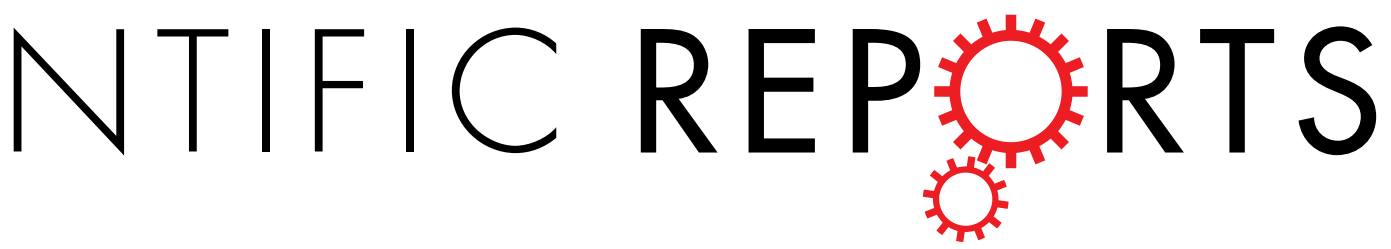

\title{
Nanogels of dual inhibitor-modified hyaluronic acid function as a potent inhibitor of amyloid $\beta$-protein aggregation and cytotoxicity
}

Zhiqiang Jiang ${ }^{1}$, Xiaoyan Dong ${ }^{1}$, Xin Yan ${ }^{1}$, Yang Liu ${ }^{2}$, Lin Zhang ${ }^{1} \&$ Yan Sun ${ }^{1}$

Inhibition of amyloid $\beta$-protein (A $\beta$ ) aggregation is considered as a promising strategy for the prevention and treatment of Alzheimer's disease. Epigallocatechin-3-gallate (EGCG) and curcumin have been recognized as effective inhibitors of $A \beta$ aggregation. Herein, we proposed dual-inhibitor modification of hyaluronic acid (HA) to explore the synergistic effect of the two inhibitors. EGCGmodified HA (EHA) formed dispersed hydrogel structures, while EGCG-curcumin bi-modified HA (CEHA) self-assembled into nanogels like curcumin-modified HA (CHA). Thioflavin T fluorescent assays revealed that the inhibitory effect of CEHA was $69 \%$ and $55 \%$ higher than EHA and CHA, respectively, and cytotoxicity assays showed that the viability of SH-SY5Y cells incubated with $A \beta$ and CEHA was $28 \%$ higher than that with $\mathrm{A} \beta$ and the mixture of EHA and $\mathrm{CHA}$. These results clearly indicate the synergism of the two inhibitors. It is considered that the difference in the hydrophobicities of the two inhibitors made the bi-modification of HA provide a favorable CEHA nanostructure that coordinated different inhibition effects of the two inhibitors. This research indicates that fabrication of dualinhibitor nanosystem is promising for the development of potent agents against $A \beta$ aggregation and cytotoxicity.

Alzheimer's disease (AD), the main kind of dementia, is an irreversible devastating neurodegenerative disorder characterized by a progressive loss of memory and other cognitive abilities ${ }^{1,2}$. Although the precise pathogenesis of $\mathrm{AD}$ is still uncertain, investigations in the past decades have put out several pathological hypotheses ${ }^{3-5}$. Among them, amyloid hypothesis is widely accepted, and it is believed that the abnormal accumulation, aggregation and deposition of amyloid $\beta$-protein $(\mathrm{A} \beta)$ contribute to the cerebral extracellular amyloid plaques that cause neurotoxicity ${ }^{6-8}$. Thus, inhibition of $\mathrm{A} \beta$ aggregation is considered as a promising strategy to fight against $\mathrm{AD}$.

Till now, many different kinds of $A \beta$ aggregation inhibitors have been reported, including small molecules ${ }^{9-11}$, peptides $^{12,13}$, antibodies ${ }^{14}$ and nanoparticles (NPs) ${ }^{15-17}$. Most of the inhibitors work by binding or adsorbing $A \beta$, which affects the conformational changes of $\mathrm{A} \beta$ and/or blocking the protein interactions. Of the inhibitors, natural polyphenols such as epigallocatechin-3-gallate (EGCG) and curcumin have received extensive studies because they show safety and high efficiency in the inhibition of $A \beta$ aggregation ${ }^{18-20}$. EGCG and curcumin have similar structures (Fig. S1 in Supplementary Materials) and their inhibitory mechanisms are similar to some degree. Their aromatic rings can interact with the aromatic residues in amyloidogenic proteins, preventing the $\pi-\pi$ interaction and blocking the aggregation process ${ }^{21,22}$. Several literatures reported that both of them could change the pathway of $A \beta$ aggregation and remodel $A \beta$ fibrils ${ }^{23,24}$. However, there exist some differences between the effects of EGCG and curcumin on $A \beta$ aggregation. It was reported that EGCG could directly bind to unfolded $A \beta$ and promote the formation of unstructured non-toxic oligomers ${ }^{25}$, while curcumin could bind to $A \beta$ due to its unique structure with two terminal phenyl groups and a rigid linker region between 8 and $16 \AA$ in length ${ }^{26}$. EGCG could bind to the 12 important residues of $\mathrm{A} \beta_{42}$ (Phe4, Arg5, Phe19, Phe20, Glu22, Lys28, Gly29, Leu34-Gly37, and Ile41) and also form hydrogen bond with $\mathrm{A} \beta^{27}$, while curcumin was reported to bind to 12 th and 17 th to 21 st

${ }^{1}$ Department of Biochemical Engineering and Key Laboratory of Systems Bioengineering of the Ministry of Education, School of Chemical Engineering and Technology, Tianjin University, Tianjin, 300072, China. ${ }^{2}$ Department of Biology \& Guangdong Provincial Key Laboratory of Marine Biotechnology, College of Science, Shantou University, Shantou, Guangdong, 515063, China. Correspondence and requests for materials should be addressed to X.D. (email: d_xy@tju.edu.cn)orY.S. (email:ysun@tju.edu.cn) 
residues of $A \beta_{42}{ }^{28}$. Some researchers believed that curcumin could only bind to the fibrillar conformation, but not to shorter, aggregation-incompetent $\mathrm{A} \beta$ fragments ${ }^{29}$. These literature results suggest that EGCG and curcumin are both effective inhibitors of $A \beta$ aggregation, but they work differently. Thus, it is speculated that there may be synergistic effects of these two inhibitors when they are put together in a nanosystem. Thus, this work was designed to fabricate a dual-inhibitor nanosystem with EGCG and curcumin to utilize their different effects and explore their potential synergistic effects.

It has been known that conjugating curcumin to the highly hydrophilic hyaluronic acid (HA) can increase curcumin solubility, and the curcumin-HA (CHA) conjugates can self-assemble into NPs via hydrophobic interactions mediated by the conjugated curcumin molecules ${ }^{30,31}$. In our previous work ${ }^{32}$, we have shown that CHA nanogels showed a size-tunable property depending on the substitution degree $(\mathrm{SD})$ of curcumin $\left(\mathrm{SD}_{\mathrm{C}}\right)$. More importantly, it turned out that CHA inhibited $\mathrm{A} \beta$ fibrillogenesis and mitigated the amyloid cytotoxicity more efficiently than free curcumin, and that there existed an optimal $\mathrm{SD}_{\mathrm{C}}$, at which the nanogels exhibited the best inhibitory effect. Based on the findings, a mechanistic CHA model was proposed. It suggested that besides the inhibitory effect of the conjugated curcumin, CHA nanogels provided additional functions: (1) the hydrogel network provided an isolation effect that could hinder the interactions between $A \beta$ molecules; (2) hydrophobic binding of $A \beta$ to curcumin and electrostatic repulsion between the bound $A \beta$ and like-charged HA could stretch the conformation of $A \beta$ monomers, thus leading to off-pathway aggregations. The latter was referred to as hydrophobic binding and electrostatic repulsion (HyBER) effect.

However, the research has revealed that CHA showed moderate increase in the inhibition effect as compared to free curcumin, and the inhibitory potency relied on whether the nanostructure of CHA was suitable for both isolation and HyBER effects. So, CHA of high SD showed lower inhibitory effect because its nanostructure was too compact for $\mathrm{A} \beta$ molecules to enter ${ }^{32}$. In order to address this issue, we have herein proposed to introduce a hydrophilic polyphenol inhibitor, EGCG, into the system to decrease the total hydrophobicity of the conjugate. It is expected that the different hydrophobic properties of EGCG and curcumin ${ }^{33,34}$ could help tune the nanostructures of the conjugates to reach more favorable isolation and HyBER effects. In addition to the improvement in nanostructure, it is also expected that the dual-inhibitor system could enhance the inhibitory effect on $A \beta$ aggregation by incorporating the potential synergistic effects of EGCG and curcumin described above. In this article, we first presented the effect of EGCG-HA (EHA) as a new example of the inhibitor-HA conjugate system. Then, the bi-modified HA with EGCG and curcumin (CEHA) with a series of SD values of EGCG and curcumin was studied to explore the best combination of the two inhibitors. The nanostructure effects on $\mathrm{A} \beta$ aggregation were then studied. The bi-modified and mono-modified conjugates were compared and the synergistic effects of EGCG and curcumin on CEHA were discussed to provide insight into its working mechanism.

\section{Results}

Characterization of EHA and CEHA conjugates. The reactions for the synthesis of EHA and CEHA conjugates are illustrated in Fig. S1 in the Supplementary Materials. The purity of CEHA conjugate was $\geq 96 \%$, as determined by reversed-phase high-performance liquid chromatography (RP-HPLC) (Fig. S2). The chemical structures of CEHA conjugates were analyzed using ${ }^{1} \mathrm{H}-\mathrm{NMR}$ (Fig. S3) and Fourier transform infrared spectroscopy (FTIR) (Fig. S4). The characteristic peaks of HA appeared at the range of methyl adjacent to carbonyl $\left(\delta=1.85 \mathrm{ppm},\left[-\mathrm{COCH}_{3}\right]\right)$ and methylene/methine $(\delta=3.2-3.8 \mathrm{ppm})$ (Fig. S3a). Successful synthesis of CEHA was confirmed by the peaks appearing from methylene and methine adjacent to benzene ring in EGCG $(\delta=2.8-$ $2.9 \mathrm{ppm}, 2 \mathrm{H}, \mathrm{PH}-\mathrm{CH}_{2}-\mathrm{CO}$ and $\left.\delta=5.45 \mathrm{ppm}, 1 \mathrm{H}, \mathrm{PH}-\mathrm{CH}-\mathrm{O}\right), \mathrm{OCH}_{3}$ groups in curcumin $(\delta=3.82 \mathrm{ppm})$, the benzene ring in both EGCG and curcumin $(\delta=6.5-7.1 \mathrm{ppm})$ and hydrogen atom adjacent to benzene ring and double bond in curcumin $(\delta=7.60 \mathrm{ppm}, 1 \mathrm{H}, \mathrm{PH}-\mathrm{CH}=)$ (Fig. S3d). From the FTIR, some specific bonds and groups were identified at the wavenumber $\left(\mathrm{cm}^{-1}\right)$ of 600-900 for benzene rings, 1300-1350 for C-O stretching frequency of ester linkage, 2100 broad band and 2800-3000 peaks for curcumin unsaturated hydrogen and the changes of band at 1100 and 3600 for the phenols (Fig. S4).

Substitution degrees of EGCG $\left(\mathrm{SD}_{\mathrm{E}}\right)$ were determined by measuring absorbance at $280 \mathrm{~nm}$ using a calibration curve (Fig. S5a,b). EHA has a similar spectrum as that of EGCG, so it was reasonable to assume that EGCG was conjugated onto the HA backbone considering HA has no absorbance at all in the wavelength range. The red shift of EHA was caused by the auxochromic effect of numerous hydroxyl groups and the ester linkage ${ }^{30,31}$. $\mathrm{SD}_{\mathrm{E}}$ values of the three synthesized EHAs were 1.67, 4.63 and 12.70 (Table S1).

EGCG and curcumin are both polyphenol inhibitors of $A \beta$ aggregation but have different hydrophobicities $^{33,34}$. We have shown that CHA conjugates formed stable nanoparticles ${ }^{32}$. By contrast, dynamic light scattering analyses (Fig. S6) and transmission electron microscopy observations (Fig. S7) revealed that EHA formed less single NPs but more large dispersed hydrogels. This is considered due to the lower hydrophobicity of EGCG than curcumin. Therefore, introduction of curcumin to EHA led to the synthesis of CEHA that formed stable NPs (Fig. S8).

Three CEHAs at each $\mathrm{SD}_{\mathrm{E}}$ were then synthesized (Table $\mathrm{S} 2$ ). The $\mathrm{SD}_{\mathrm{C}}$ values of the CEHAs were determined by measuring the absorbance at $440 \mathrm{~nm}$ using a calibration curve (Fig. S5c,d). The hydrodynamic size (diameters) of the CEHAs in the PBS solution decreased as $\mathrm{SD}_{\mathrm{C}}$ or $\mathrm{SD}_{\mathrm{E}}$ increased (Fig. 1a). The mean sizes of the CEHAs were more sensitive to $\mathrm{SD}_{\mathrm{C}}$ than to $\mathrm{SD}_{\mathrm{E}}$, due to the higher hydrophobicity of curcumin. There was no discernible difference in the zeta potentials of CEHAs as $\mathrm{SD}_{\mathrm{C}}$ changed (Fig. 1b).

In the following discussion, the conjugate concentration used below for EHA, CHA or CEHA is described by the concentration of inhibitor, EGCG or curcumin, in the conjugate, to evaluate the conjugate inhibitory effect and compare with free inhibitors. Namely, the EGCG/curcumin concentration in the supplied CEHA was adjusted to match the concentration of the specific free molecule in solution. 

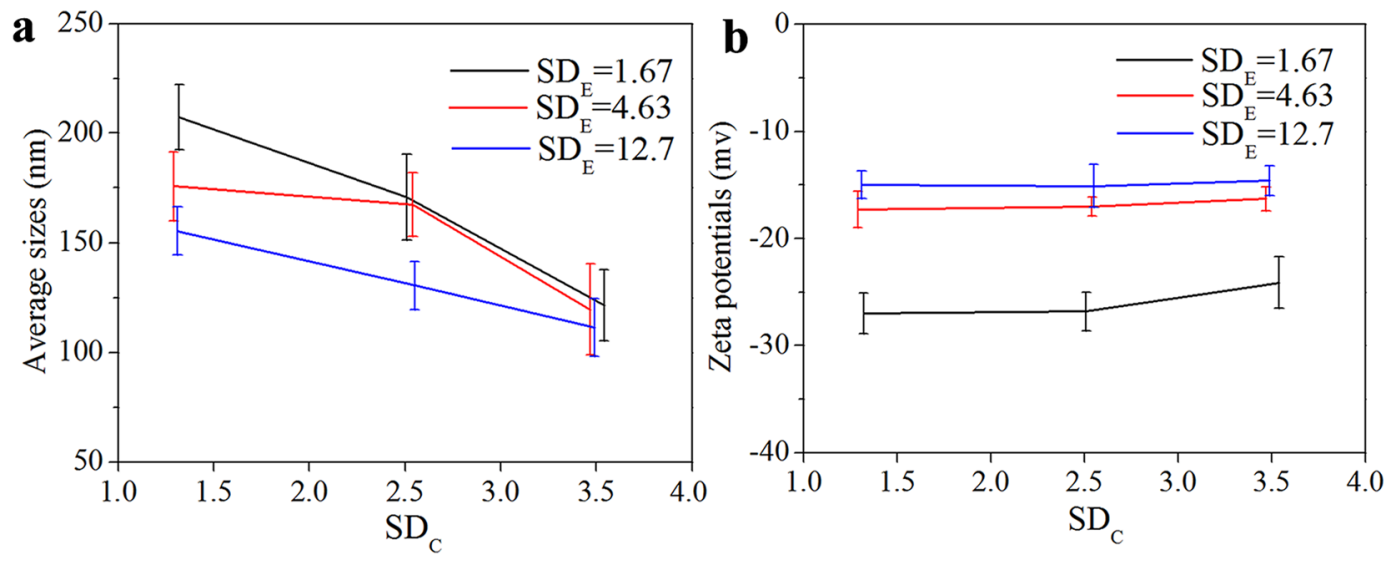

Figure 1. Average hydrodynamic sizes (a) and zeta potentials (b) of CEHA NPs at different $\mathrm{SD}_{\mathrm{E}}$ as a function of $\mathrm{SD}_{\mathrm{C}}$ in the $\mathrm{PBS}$ solution at $37^{\circ} \mathrm{C}$. The data were the average values of the data determined with three samples and the error bars represent the standard deviations.

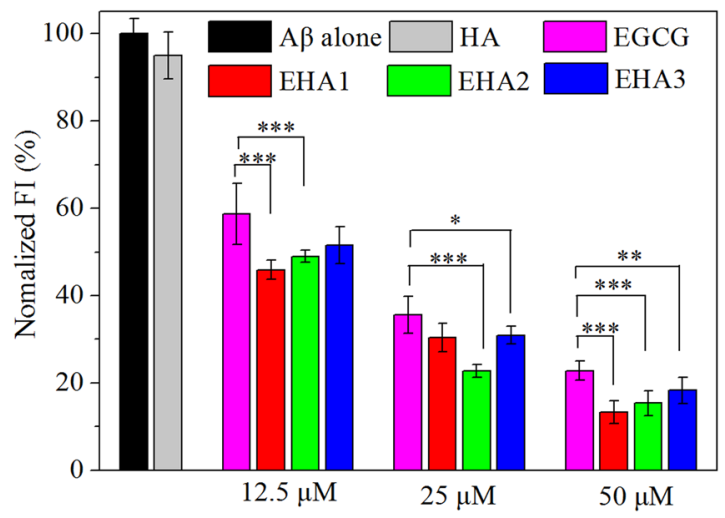

Figure 2. ThT fluorescence intensities of $A \beta_{42}(25 \mu \mathrm{M})$ incubated for $48 \mathrm{~h}$ with HA, free EGCG and the three EHAs of different EGCG $\mathrm{SD}_{\mathrm{E}}$ values. HA concentration was $2 \mathrm{mg} / \mathrm{mL}$. EHA concentration is expressed as the equivalent concentration of EGCG carried in the conjugate. Each condition of incubation was conducted in triplicate in three Eppendorf tubes and the average value is reported with standard deviation. $* * * p<0.001$, $* * \mathrm{p}<0.01$ and $* \mathrm{p}<0.05$ compared to free EGCG groups.

Elevated inhibitory effect of EHA on $\mathbf{A} \boldsymbol{\beta}_{\mathbf{4 2}}$ aggregation. Figure 2 shows that HA had no remarkable influence on the thioflavin T fluorescent intensity (ThT FI) of $A \beta_{42}$ at high concentrations. However $A \beta_{42}$ incubated with EHA hydrogels had slightly lower ThT FI value than that incubated with free EGCG at the same EGCG concentration, demonstrating that the inhibitory effect of EGCG on $\mathrm{A} \beta_{42}$ aggregation was improved by conjugating onto HA. It also shows a dose-dependent effect of EHAs on $A \beta_{42}$ aggregation. The results demonstrate that the HA modification improved the inhibitory effect of the polyphenol on $\mathrm{A} \beta_{42}$ aggregation, and it is considered that the CHA model proposed previously also works to some extent for the dispersed EHA hydrogel structures ${ }^{32}$.

Elevated inhibitory effect of CEHA on $\mathbf{A} \beta_{42}$ aggregation. ThT FI of $\mathrm{A} \beta_{42}$ aggregation was investigated to examine the inhibitory effect of CEHA. At first, EGCG concentration was fixed at $5 \mu \mathrm{M}$ and curcumin concentration varied from 0.5 to $10.6 \mu \mathrm{M}$ for the first nine CEHA nanogels listed in Table S2 (CEHA mass concentration varied from 0.016 to $0.12 \mathrm{mg} / \mathrm{mL}$ for the different CEHAs to keep EGCG concentration unchanged). The ThT FI values of $A \beta_{42}$ incubated with the nine CEHAs for $48 \mathrm{~h}$ are shown in Fig. 3, and the control groups were set as the free EGCG-curcumin mixture with the same EGCG/curcumin concentrations as the corresponding CEHA group. The figure over a column represents the reducing percentage (RP) of ThT FI for the CEHA as compared to that for the free EGCG-curcumin mixture. In each subfigure in Fig. 3, the CEHAs had the same $\mathrm{SD}_{\mathrm{E}}$ but different $\mathrm{SD}_{\mathrm{C}}$ values. Clearly, CEHAs showed higher inhibitory effect than the free inhibitor mixtures, because they decreased the ThT FI of $\mathrm{A} \beta_{42}$ aggregation by over $20 \%$ to $50 \%$ as compared to the free EGCG-curcumin groups. In general, the $\mathrm{RP}$ decreased with increasing $\mathrm{SD}_{\mathrm{E}}$ as well as $\mathrm{SD}_{\mathrm{C}}$. For example, the RP for CEHA1-3 decreased from $56.5 \%$ to $46.7 \%$ with the increase in $\mathrm{SD}_{\mathrm{C}}$. It is considered that introducing more curcumin into the EHA backbone resulted in more compact nanostructure of CEHA, similar to the case of $\mathrm{CHAs}^{32}$, which compromised the inhibitory effect of the nanogels. By judging from the RP values, it is obvious that CEHA1-3 with the lowest $\mathrm{SD}_{\mathrm{E}}$ (Table S2) were more efficient than the other six, indicating that $\mathrm{SD}_{\mathrm{E}}$ was also important in influencing the CEHA nanostructure that contributed to the inhibitory effect of the CEHA conjugates. 

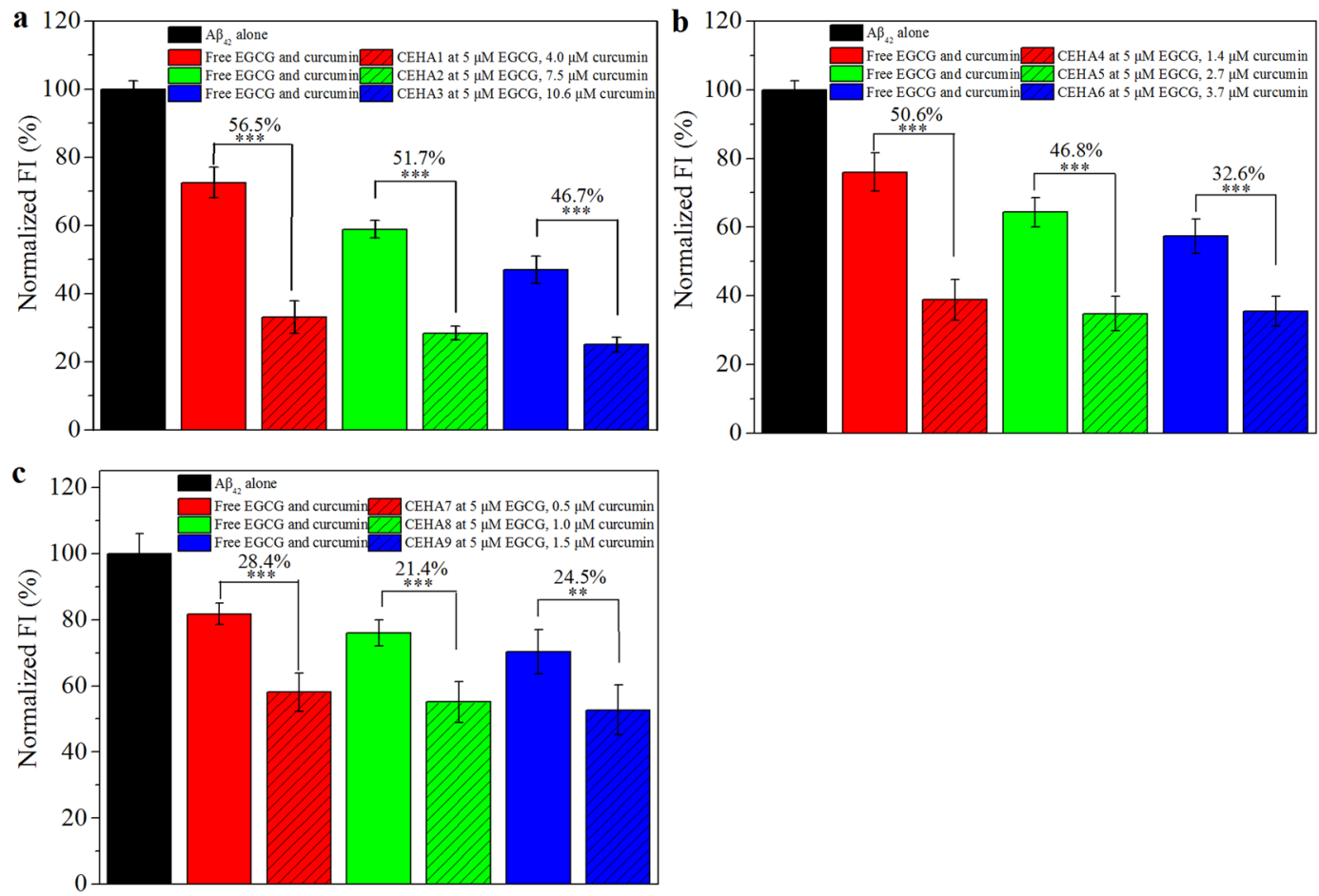

Figure 3. ThT FI of $A \beta_{42}$ incubated for $48 \mathrm{~h}$ with different CEHAs by comparison with the mixture of free EGCG-curcumin at the same inhibitor concentrations at each group. CEHA concentration is expressed as the equivalent concentration of free EGCG and curcumin carried in the conjugates (CEHA mass concentrations were $0.016-0.12 \mathrm{mg} / \mathrm{mL}$ ). EGCG concentration was fixed at $5 \mu \mathrm{M}$ with varying curcumin concentrations. (a) CEHA1-3 $\left(\mathrm{SD}_{\mathrm{E}}=1.67\right)$; (b) CEHA4-6 $\left(\mathrm{SD}_{\mathrm{E}}=4.63\right)$; (c) CEHA7-9 $\left(\mathrm{SD}_{\mathrm{E}}=12.7\right)$. Each condition of incubation was conducted in triplicate in three Eppendorf tubes and the average value is reported with standard deviation. The figures in percentage above the columns stand for the RPs of the ThT FI for the CEHA groups (slash bars) as compared to those for the corresponding free EGCG-curcumin groups (solid bars). $* * * p<0.001$ and $* * \mathrm{p}<0.01$ compared to the free inhibitor groups.

When fixing curcumin concentration at $5 \mu \mathrm{M}$ with varying EGCG concentrations $(2.4-48 \mu \mathrm{M})$ for CEHA (CEHA mass concentration varied from 0.06 to $0.15 \mathrm{mg} / \mathrm{mL}$ for the different CEHAs to keep curcumin concentration unchanged), similar results were obtained (Fig. S9). However, in comparison of Fig. S9 with Fig. 3, it is clear that the RPs at lower curcumin and higher EGCG concentrations (Fig. S9) were much lower than those at lower EGCG and higher curcumin concentrations (Fig. 3). This indicates that the enhancing effect of the bi-conjugation of HA become less significant for the combination with higher EGCG concentrations.

The aggregation morphologies of $A \beta_{42}$ incubated with different inhibitor systems were observed with atomic force microscopy (AFM). Figure 4a shows that $A \beta_{42}$ alone formed mature fibrils of several micrometers in length. Incubation with free EGCG and curcumin mixture resulted in fewer fibrous aggregates (Fig. 4b). However, it is seen that few fibrils but spherical aggregates were observed in the presence of CEHA1 (Fig. 4c), indicating that the CEHA nanogels altered the structure and morphologies of $\mathrm{A} \beta_{42}$ aggregates.

Finally, cell viability assays were performed to examine the detoxification effect of CEHA nanogels. EGCG was little cytotoxic but curcumin was slightly toxic at $25 \mu \mathrm{M}$ (Fig. S10a). In comparison, little cytotoxicity of the nine CEHA nanogels at $25 \mu \mathrm{M}$ curcumin was observed (Fig. S10b), suggesting the biocompatibility of the CEHA nanogels.

Two CEHA conjugates, CEHA1 and CEHA4, which showed better inhibition performance (lower FIs and higher RPs) as evidenced in Fig. 3, were chosen for cell viability assays. As shown in Fig. 5, the cell viability in the group treated with PBS was set as $100 \%$. CEHA1 (containing $5 \mu \mathrm{M}$ EGCG and $4 \mu \mathrm{M}$ curcumin) improved cell viability by $43.5 \%$ and CEHA4 (containing $5 \mu \mathrm{M}$ EGCG and $1.4 \mu \mathrm{M}$ curcumin) improved cell viability by $23.8 \%$, as compared to the corresponding free inhibitor systems. With the CEHA1 containing only $5 \mu \mathrm{M}$ EGCG and $4 \mu \mathrm{M}$ curcumin, the cell viability increased to $89 \%$, about $77 \%$ higher than the $\mathrm{A} \beta_{42}$ alone group (cell viability, $50.5 \%$ ). CEHA1 presented higher performance than CEHA4. Higher curcumin concentration in CEHA1 might be part of the cause, but considering that such a low curcumin concentration does not provide significant inhibition effect ${ }^{35,36}$, difference in the nanostructures of the two nanogels was most likely to be responsible for the significant effect on the cell viability results.

Nanostructure effect. Thus, to better understand the relationship between the nanostructure and the inhibitory effect, we synthesized two more CEHA conjugates, CEHA10 and CEHA11 (Table S2). CEHA1, CEHA6, CEHA10 and CEHA11 have almost the same $\mathrm{SD}_{\mathrm{E}} / \mathrm{SD}_{\mathrm{C}}$ ratio $(1.30 \pm 0.04)$. Thus, we can design an inhibition experiment with these four CEHAs containing both EGCG and curcumin at the same concentrations (the mass concentration of CEHAs varied from 0.04 to $0.12 \mathrm{mg} / \mathrm{mL}$ to keep EGCG and curcumin concentrations 

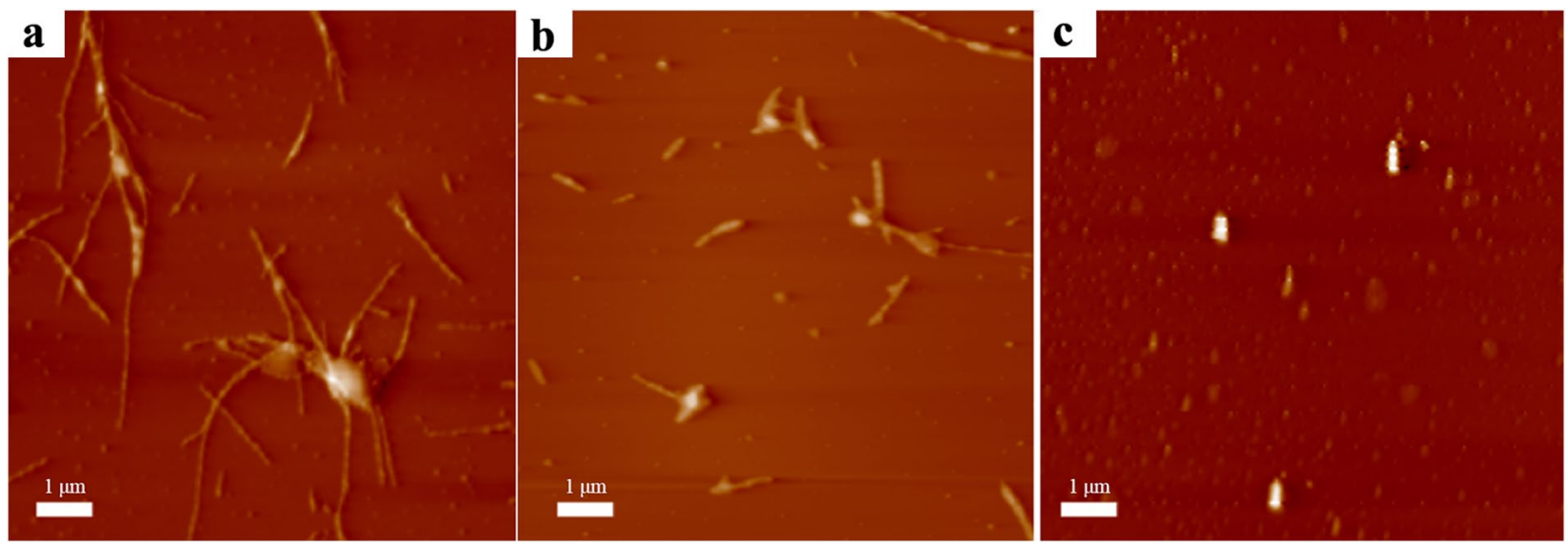

Figure 4. The AFM images of $A \beta_{42}$ incubated for $48 \mathrm{~h}$. (a) $25 \mu \mathrm{MA} \beta_{42}$ alone; (b) $25 \mu \mathrm{M} \mathrm{A} \beta_{42}$ with a mixture of $5 \mu \mathrm{M}$ EGCG and $4 \mu \mathrm{M}$ curcumin; (c) $25 \mu \mathrm{M} \mathrm{A} \beta_{42}$ with $0.12 \mathrm{mg} / \mathrm{mL}$ CEHA1 nanogel (containing $5 \mu \mathrm{M}$ EGCG and $4 \mu \mathrm{M}$ curcumin).

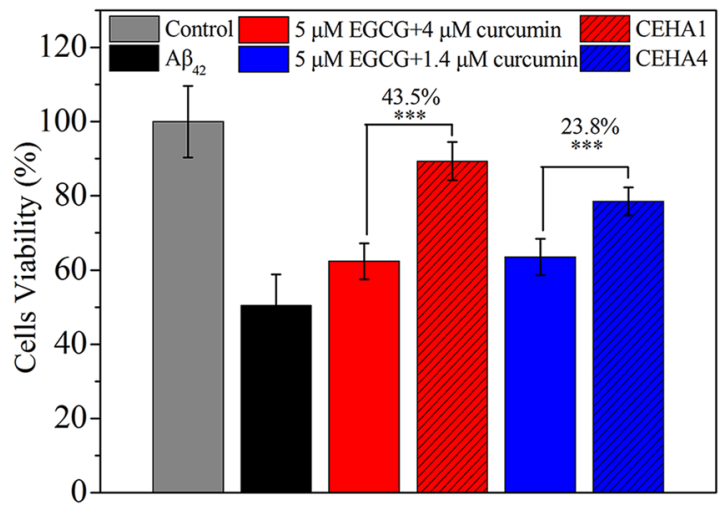

Figure 5. Cell viability of SH-SY5Y cells incubated with $A \beta_{42}$ aggregates obtained by pre-incubation of $A \beta_{42}$ with free EGCG-curcumin groups (solid bars) or CEHA (slash bars). All the samples had $5 \mu \mathrm{M}$ EGCG. Each CEHA group contained the same EGCG and curcumin concentrations as the corresponding free EGCGcurcumin group. Each condition was conducted in sextuplicate in six wells of 96 -well plate and the average value is reported with standard deviation. $* * * \mathrm{p}<0.001$ compared to the free inhibitor groups.

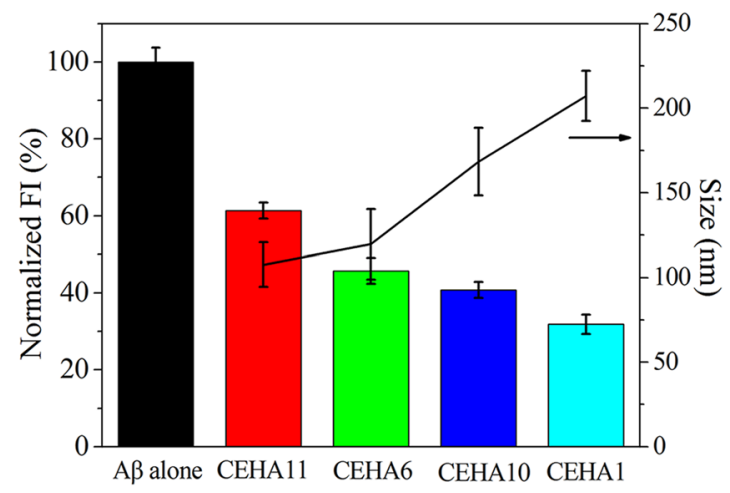

Figure 6. ThT FI of $A \beta_{42}$ incubated for $48 \mathrm{~h}$ with the four $\mathrm{CEHAs}$ with almost the same $\mathrm{SD}_{\mathrm{E}} / \mathrm{SD}_{\mathrm{C}}$ ratio $(1.3 \pm 0.04)$. All CEHAs contains $5 \mu \mathrm{M}$ EGCG and $3.8 \pm 0.2 \mu \mathrm{M}$ curcumin. Each condition of incubation was conducted in triplicate in three Eppendorf tubes and the average value is reported with standard deviation.

unchanged) (Fig. 6). The average hydrodynamic size (diameter) of the CEHAs increased as total SD decreased (CEHA1 < CEHA10 < CEHA6 < CEHA11 in total SD), because the nanostructures became less compact at lower total SD values as discussed above. Interestingly, the ThT FI of $A \beta_{42}$ incubated with the CEHAs also decreased as 

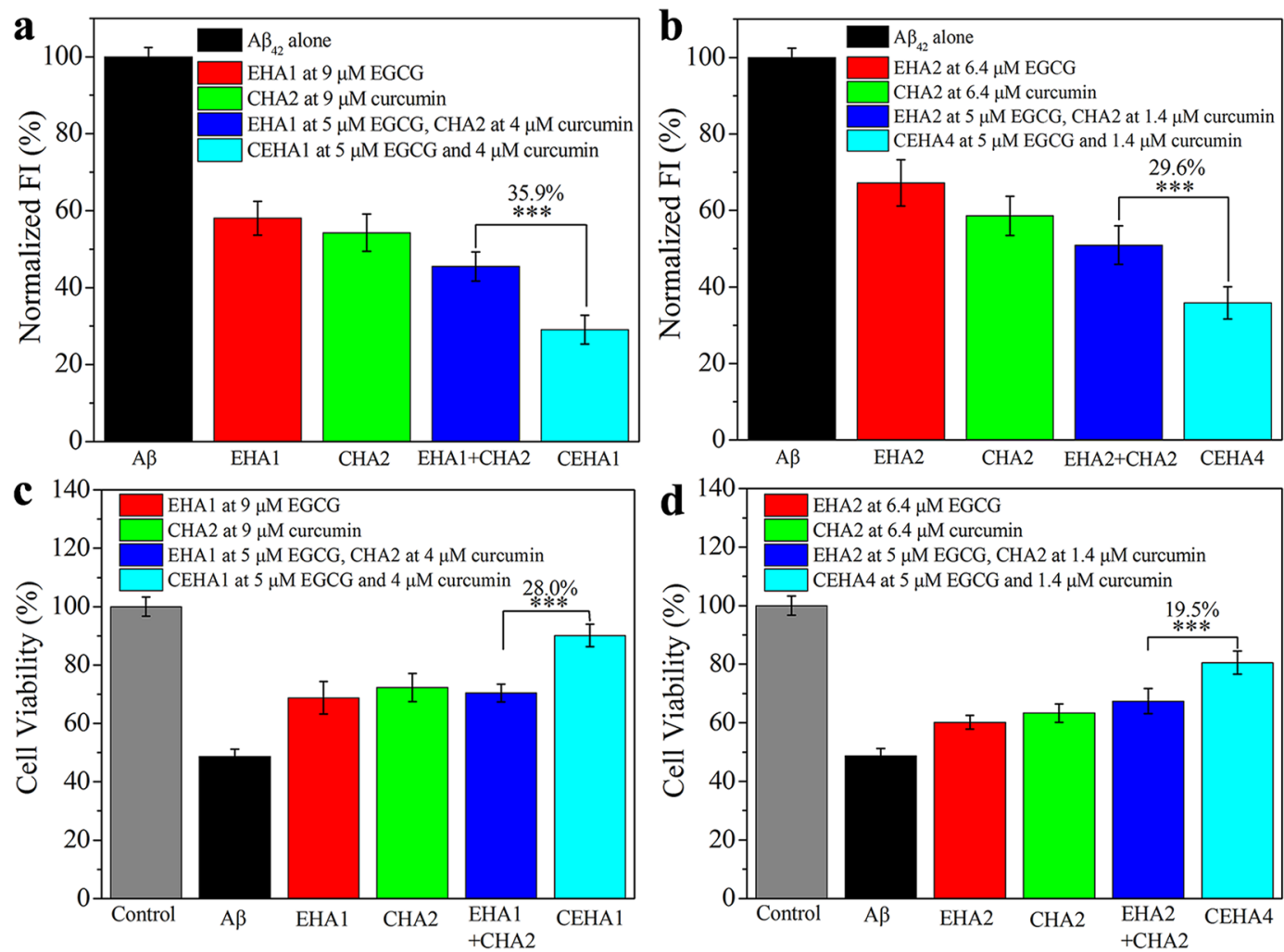

Figure 7. (a,b) Comparison of the ThT FI values of $A \beta_{42}(25 \mu \mathrm{M})$ incubated for $48 \mathrm{~h}$ with EHA, CHA, mixture of EHA and CHA, and CEHA at the same total conjugated polyphenol concentration $9 \mu \mathrm{M}$ in (a) and $6.4 \mu \mathrm{M}$ in (b). Each condition of incubation was conducted in triplicate in three Eppendorf tubes and the average value is reported with standard deviation. (c,d) Cell viability of SH-SY5Y cells incubated with $A \beta_{42}$ aggregates obtained by pre-incubation of $\mathrm{A} \beta_{42}$ with EHA, CHA, mixture of EHA and CHA and CEHA at the same total conjugated polyphenol concentration $9 \mu \mathrm{M}$ in $(\mathbf{c})$ and $6.4 \mu \mathrm{M}$ in (d). Each condition was conducted in sextuplicate in six wells of 96-well plate and the average value is reported with standard deviation. (a) and (c) Comparison of EHA1, CHA2, mixture of EHA1 $\left(\mathrm{SD}_{\mathrm{E}}=1.67\right)$ and $\mathrm{CHA} 2\left(\mathrm{SD}_{\mathrm{C}}=1.32\right)$, and CEHA1 with the same $\mathrm{SD}_{\mathrm{E}}$ as EHA1 and $\mathrm{SD}_{\mathrm{C}}$ as CHA2; (b) and (d) Comparison of EHA2, CHA2, mixture of EHA2 $\left(\mathrm{SD}_{\mathrm{E}}=4.63\right)$ and $\mathrm{CHA} 2$ $\left(\mathrm{SD}_{\mathrm{C}}=1.32\right)$, and CEHA4 with the same $\mathrm{SD}_{\mathrm{E}}$ as EHA2 and $\mathrm{SD}_{\mathrm{C}}$ as CHA2. $* * * \mathrm{p}<0.001$ compared to the monomodified EHA and CHA mixture.

the average CEHA hydrodynamic size increased (Fig. 6). For example, CEHA1 reduced the ThT FI about $48.6 \%$ more than CEHA11 did. Since EGCG and curcumin concentrations ( $5 \mu \mathrm{M}$ EGCG and $3.8 \pm 0.2 \mu \mathrm{M}$ curcumin) were almost the same for the four conjugates, this clearly indicates that the CEHA nanostructure significantly influenced the inhibitory effect of the conjugates. It is considered that in the four conjugates, CEHA1 had the most suitable SD that led to the formation of the most favorable nanostructure for $A \beta_{42}$ to enter into and to interact with the inhibitors. This allowed CEHA1 to make full use of the conjugated inhibitors and coordinated the isolation and HyBER effects ${ }^{32}$ to achieve the highest inhibitory effect among the four conjugates.

Synergistic effect. The mono-modified EHAs and CHAs both improved the effect of the polyphenol inhibitors based on the self-assembled structures of the conjugates. Considering that EGCG and curcumin have different hydrophobicities ${ }^{33,34}$ and different inhibition mechanisms in molecular level ${ }^{25-29}$, it is expected that a bi-modified CEHA with a suitable EGCG/curcumin ratio could form a favorable nanostructure for inhibiting $\mathrm{A} \beta_{42}$ aggregation and display a synergistic effect of EGCG and curcumin.

To verify the synergistic effect, we compared the difference between a bi-modified CEHA and a mono-modified EHA or CHA at the same (total) inhibitor concentrations. Figure 7a shows the ThT FI data of $\mathrm{A} \beta_{42}$ incubated with EHA, CHA, the mixture of EHA and CHA, and CEHA at a total inhibitor concentration of $9 \mu \mathrm{M}$. It is clear that $\mathrm{A} \beta_{42}$ incubated with CEHA1 $\left(\mathrm{SD}_{\mathrm{E}}=1.67, \mathrm{SD}_{\mathrm{C}}=1.32\right)$ displayed the lowest FI, and its FI was $35.9 \%$ lower than that with the mixture of EHA1 and CHA2. CEHA4 showed the same tendency (Fig. 7b). Cell viability assays at the same (total) inhibitor concentrations exhibited similar results: the cell viability with CEHA1 was $28.0 \%$ higher than that with the mixture of EHA1 and CHA2, and the cell viability with CEHA4 was $19.5 \%$ higher than that with the mixture of EHA2 and CHA2 (Fig. 7c,d). CEHA1 and CEHA4 increased the cell viability to $90.1 \%$ and $80.5 \%$, respectively, which were respectively $85.1 \%$ and $65.4 \%$ higher than the $A \beta_{42}$ alone group.

The synergistic effect of conjugated EGCG and curcumin in CEHAs on inhibiting $A \beta_{42}$ aggregation was further studied by ThT fluorescent assays. Here, we introduced a synergy factor, $S F$, defined as ${ }^{37}$ : 

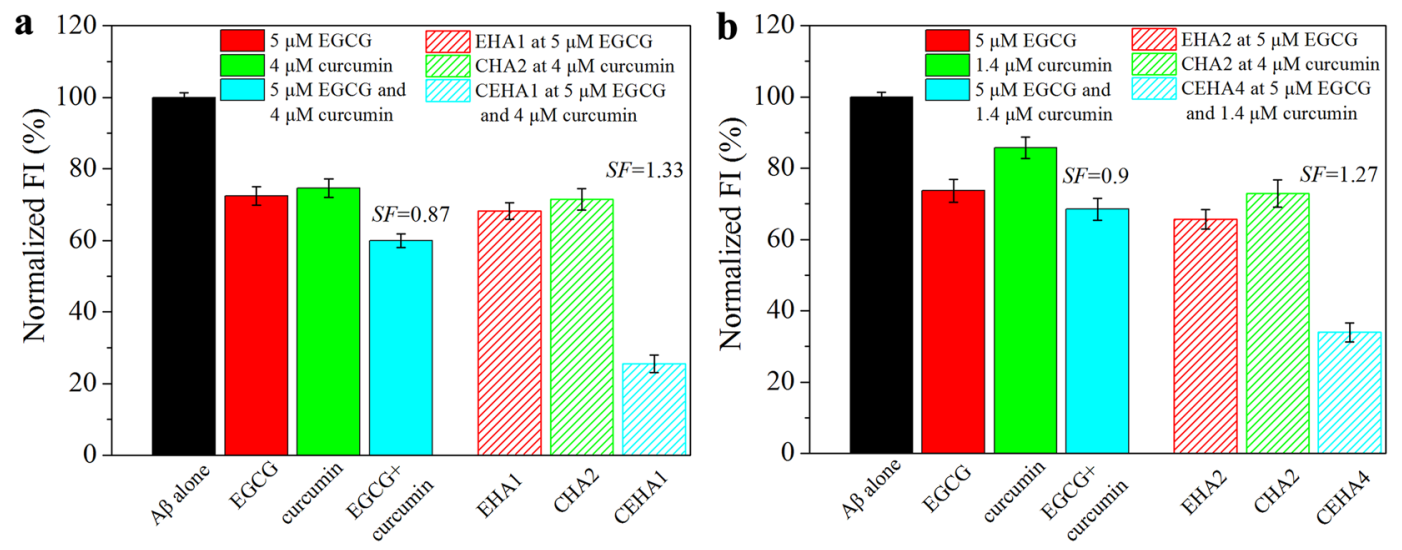

Figure 8. (a) Determination of the $S F$ value for CEHA1: ThT FI of $A \beta_{42}$ alone and incubated with EHA1 at $5 \mu \mathrm{M}$ EGCG (red slash bars), CHA2 at $4 \mu \mathrm{M}$ curcumin (green slash bars) and CEHA1 at $5 \mu \mathrm{M}$ EGCG and $4 \mu \mathrm{M}$ curcumin (cyan slash bars). (b) Determination of the $S F$ value for CEHA4: ThT FI of $A \beta_{42}$ alone and incubated with EHA2 at $5 \mu \mathrm{M}$ EGCG (red slash bars), CHA2 at $1.4 \mu \mathrm{M}$ curcumin (green slash bars) and CEHA 4 at $5 \mu \mathrm{M}$ EGCG and $1.4 \mu \mathrm{M}$ curcumin (cyan slash bars). $\mathrm{A} \beta_{42}$ incubated with free EGCG and curcumin at the same concentrations are also reported as controls in the two figures. Each condition of incubation was conducted in triplicate in three Eppendorf tubes and the average value is reported with standard deviation.

$$
S F=\frac{100 \%-R_{A B}}{100 \%-R_{A} \times R_{B}}
$$

where $R_{\mathrm{A}}$ and $R_{\mathrm{B}}$ are the remaining ThT FI for the mono-inhibitor system and $R_{\mathrm{AB}}$ is the remaining ThT FI for the dual-inhibitor system. $S F>1$ means a synergism of the two inhibitors, whereas $S F<1$ means antagonism of the two inhibitors.

The $S F$ values for the two representative dual-inhibitor conjugates, CEHA1 and CEHA4, were determined by ThT fluorescent assays (Fig. 8) to be 1.33 and 1.27, respectively. As controls, free EGCG-curcumin showed an antagonistic effect with an $S F$ value of about 0.9 . The results indicate that only the conjugated EGCG and curcumin in the CEHA nanogels had significant synergistic functions. In other words, the dual-conjugation onto HA was the cause for the synergistic effect.

Effects of CEHA on the kinetics of $A \beta_{40}$ aggregation was studied by measuring the aggregation dynamics. The growth curves were normalized using a sigmoidal fit equation ${ }^{38}$,

$$
y=y_{0}+\frac{y_{\max }-y_{0}}{1+\exp \left(-\left(t-t_{1 / 2}\right) k\right)}
$$

where $y$ is the ThT FI at time $t, y_{0}$ and $y_{\max }$ are the minimum and maximum ThT FI, respectively, $t_{1 / 2}$ is the time when the ThT FI reaches half the maximum intensity, and $k$ is the apparent first-order aggregation constant. From the fitting the lag phase time $\left(T_{\mathrm{lag}}\right)$ could be calculated from ${ }^{38}$,

$$
T_{\text {lag }}=t_{1 / 2}-2 / k
$$

Fig. S11 shows the fitting of Eq. (2) to the normalized dynamic data. The apparent first-order aggregation constant $k$ value and lag phase time $T_{\text {lag }}$ determined from Eq. (3) are listed in the Table 1. EGCG did not affect the $k$ value although it decreased the plateau ThT FI of $25 \mu \mathrm{M} \mathrm{A} \beta_{40}$ by $40 \%$ at $9 \mu \mathrm{M}$. Curcumin decreased the $k$ value slightly and prolonged the $T_{\text {lag }}$ of $25 \mu \mathrm{M} \mathrm{A} \beta_{40}$ by $40 \%$ at $9 \mu \mathrm{M}$. This further demonstrated the different working mechanisms of EGCG and curcumin. As for the nanogel (EHA, CHA or CEHA) containing the same concentration of EGCG or curcumin, it remarkably decreased the $k$ value (e.g. CEHA1 decreased $k$ from 0.68 to 0.16 ) and also prolonged the $T_{\mathrm{lag}}$. Considering that the primary nucleation rate can be reflected by the $T_{\mathrm{lag}}$ and the elongation rate and secondary nucleation rate by the $k$ value ${ }^{39}$, it is clear that the nanogels enhanced the effects of EGCG and curcumin on the elongation and secondary nucleation processes.

\section{Discussion}

It has been recognized that in on-pathway fibrillation, $A \beta$ monomers aggregate to amyloid fibrils via several metastable oligomers after transition into $\beta$-rich conformations ${ }^{40-43}$. Previous work has revealed that $\mathrm{CHA}$ nanogels might provide an isolation effect to segregate the $A \beta$ molecules and a HyBER effect that disturbed the conformation of the bound $\mathrm{A} \beta$ molecules ${ }^{32}$. These two effects were influenced by the conjugate nanostructure (Fig. 6 ). $\mathrm{SD}_{\mathrm{C}}$ is a key factor influencing the conjugate nanostructure (Fig. la). When the $\mathrm{SD}_{\mathrm{C}}$ was high, the nanostructure would become too compact, preventing $\mathrm{A} \beta$ from entering the nanogels. When the $\mathrm{SD}_{\mathrm{C}}$ was very low, the nanostructure would become dispersed as in the case of EHA gel (Figs S6 and S7), which would weaken the isolation effect of the network. Because of the low hydrophobicity of EGCG, the EHA hydrogels should have loose structures and wide networks (Fig. S7). 


\begin{tabular}{|l|l|l|l|l|}
\hline Inhibitors & $\boldsymbol{k}\left(\mathbf{h}^{-1}\right)$ & $\boldsymbol{t}_{\mathbf{1} / 2}(\mathbf{h})$ & $\boldsymbol{T}_{\text {lag }}(\mathbf{h})$ & $\begin{array}{l}\text { Plateau ThT }^{\mathbf{b}} \\
\text { FI (\%) }\end{array}$ \\
\hline None & $0.68 \pm 0.03$ & $21.0 \pm 1.3$ & $18.5 \pm 1.2$ & $100 \pm 9.2$ \\
\hline EGCG & $0.64 \pm 0.04$ & $24.0 \pm 0.8$ & $20.9 \pm 0.6$ & $59.6 \pm 4.4$ \\
\hline Curcumin & $0.55 \pm 0.11$ & $29.0 \pm 1.6$ & $25.3 \pm 2.2$ & $75.6 \pm 4.6$ \\
\hline EGCG + Curcumin & $0.63 \pm 0.09$ & $28.3 \pm 1.6$ & $25.1 \pm 1.9$ & $80.1 \pm 7.0$ \\
\hline EHA & $0.43 \pm 0.05$ & $28.7 \pm 2.0$ & $24.0 \pm 2.5$ & $62.1 \pm 3.9$ \\
\hline CHA & $0.30 \pm 0.06$ & $37.7 \pm 1.9$ & $30.9 \pm 3.0$ & $44.0 \pm 8.8$ \\
\hline CEHA1 & $0.16 \pm 0.02$ & $40.8 \pm 2.5$ & $28.4 \pm 3.2$ & $33.8 \pm 6.9$ \\
\hline
\end{tabular}

Table 1. Kinetic parameters of $A \beta_{40}$ aggregation ${ }^{\mathrm{a}}$. ${ }^{\mathrm{a} D a t a}$ represent average values of six replica of $\mathrm{A} \beta_{40}$ aggregation dynamics and the standard deviations. ${ }^{b}$ Steady-state ThT FI values. The value without any inhibitor was set as $100 \%$ (first row) and the others were normalized by comparison to this value.

However, CEHA contained both EGCG and curcumin and the strong hydrophobicity of curcumin stabilized the nanostructures (Fig. S8). The presence of EGCG in CEHA would prevent the nanostructure from becoming too compact for $\mathrm{A} \beta$ to enter at higher SD values. In this way, conjugated EGCG could provide sufficient polyphenol inhibitor inside the nanostructure for binding $A \beta$. Thus, CEHA could not only exhibit favorable nanostructure (Fig. 6) that coordinated the isolation and HyBER effects, but also provided high conjugated inhibitor (including curcumin and EGCG) concentration that could function effectively inside the nano-conjugates. This is the synergistic effects of EGCG and curcumin in the bi-conjugated nanosystem as compared to EHA or CHA (Figs 7 and 8 and Table 1). The different hydrophobicities of EGCG and curcumin and suitable SD provided a favorable nanostructure for $\mathrm{A} \beta$ penetration and isolating them from each other. The HyBER effect of nanogels might influence the aggregation process of $\mathrm{A} \beta$ to decrease the $k$ value and prolong $T_{\text {lag }}$ (Table 1) and then enhance the effects of conjugated EGCG and curcumin on the elongation and secondary nucleation processes of $A \beta_{40}$. Consequently, the favorable nanostructure of CEHA and the synergistic effect of the two inhibitors in the nanogels led to the potent inhibition on the cytotoxicity of $A \beta$ aggregates at low total inhibitor concentrations (Figs $5,7 c, d$ ).

In conclusion, this research has designed bi-modification of HA with EGCG and curcumin to develop a potent nano-inhibitor on $A \beta$ aggregation and cytotoxicity. This design was to explore a potential synergistic effect of the two inhibitors in the conjugates because EGCG and curcumin are both effective inhibitors of $A \beta$ aggregation, but they work differently. The synergistic effect has been proved by extensive inhibition experiments and a synergy factor over 1.3 was observed. It was found that the introduction of EGCG to the curcumin-modified HA could modulate the nanostructure of the self-assembled hydrogels. Therefore, there was a suitable substitution degrees of EGCG and curcumin, at which the CEHA could not only provide sufficient conjugated polyphenol inhibitors that functioned synergistically inside the nano-conjugates, but also provide a favorable nanostructure that effectively influenced the aggregation process of $A \beta$. The research indicates that fabrication of a dual-inhibitor nanosystem is promising for the development of a potent agent against $\mathrm{A} \beta$ aggregation and cytotoxicity.

\section{Methods}

Chemicals and reagents. Curcumin (98\%), EGCG (95\%), hexafluoroisopropanol (HFIP), 3-(4,5-dimethylthiazol-2-yl)-2,5-diphenyltetrazolium bromide (MTT), thioflavin T (ThT), 1, 3-dicyclohexylcarbodiimide (DCC) and 4-dimethylaminopyridine (DMAP) were obtained from Sigma-Aldrich (St. Louis, MO, USA). Hyaluronic acid sodium salt (HA) (MW $\left.>1 \times 10^{6}, 95 \%\right)$ was purchased from Aladdin (Shanghai, China). Dimethyl sulfoxide (DMSO) was purchased from Daji Huagong (Tianjin, China). $A \beta_{42}$ and $A \beta_{40}$ were received from GL Biochem (Shanghai, China). SH-SY5Y cells were from the Cell Bank of the Chinese Academy of Sciences (Shanghai, China). Dulbecco's Modified Eagle Medium/Ham's F-12 (DMEM/F12) and fetal bovine serum (FBS) were obtained from Invitrogen (Carlsbad, CA, USA). Other chemicals were all of the highest purity available from local sources. Deionized water was used for all solution preparations.

Synthesis of EHA, CHA and CEHA conjugates. EHA and CEHA conjugates were synthesized according to the methods reported previously (Fig. S1c) ${ }^{30-32}$. Briefly, HA and EGCG/curcumin were dissolved in water/DMSO system $(1: 1 \mathrm{v} / \mathrm{v})$ with DCC and DMAP as catalysts. The reaction was carried out at $65^{\circ} \mathrm{C}$ for $6 \mathrm{~h}$, and the resulting products were collected by dialysis with a membrane of molecular weight cut off of $10-14 \mathrm{kDa}$ against DMSO for two days and then against deionized water for another three days to remove non-reacted substances and byproducts. The initial EGCG/HA in the reaction was tuned to prepare three EHA conjugates with different $\mathrm{SD}_{\mathrm{E}}$.

CEHA conjugates were synthesized from EHA conjugates by further reaction with curcumin of different concentrations under the same reaction condition described above. The products were also recovered by the same procedure.

Characterization of the conjugates. The purity of CEHA conjugates was analyzed by RP-HPLC on Agilent 1100series (Agilent Technologies, Santa Clara, CA) with a Waters C4 reversed-phase column (Waters, Milford, MA, USA) with UV-visible detections at 280 and $440 \mathrm{~nm}$. The purified CEHA conjugates were then characterized by FTIR (Nicolet 6700, Nicolet, USA) and nuclear magnetic resonance spectroscopy $\left({ }^{1} \mathrm{H}\right.$ NMR) (Varian Inova, Varian Medical System, Inc., CA, USA).

The conjugates were then characterized by UV-visible spectroscopy. The $\mathrm{SD}_{\mathrm{E}}$ and $\mathrm{SD}_{\mathrm{C}}$ in the conjugates were calculated by the absorbance measurement at $280 \mathrm{~nm}$ and $440 \mathrm{~nm}$, respectively, by using their calibration curves 
respectively prepared with free EGCG and curcumin ${ }^{29,44}$. The $\mathrm{SD}_{\mathrm{E}}$ or $\mathrm{SD}_{\mathrm{C}}$ was defined as the number of EGCG or curcumin molecules per hundred HA monomer units.

The morphologies and structures of EHA and CEHA self-assembled NPs were observed by transmission electron microscopy of model JEM-2100F (JEOL, Tokyo, Japan). The sample was prepared by dropping $10 \mu \mathrm{L}$ solutions onto a carbon-coated copper grid and air-dried. The hydrodynamic size (diameter) of CEHA was determined by dynamic light scattering (DLS) by using Zetasizer Nano (Malvern Instruments, Worcestershire, UK) at $25^{\circ} \mathrm{C}$ with the backscattered angle detection at $173^{\circ}$. The surface zeta potentials of the NPs were measured using the same equipment.

Preparation of $\mathbf{A} \boldsymbol{\beta}$ monomer solutions. $A \beta_{42}$ and $A \beta_{40}$ monomer solutions were prepared as described earlier ${ }^{32,43}$. The $A \beta_{42}$ and $A \beta_{40}$ protein sample was dissolved in HFIP and sonicated for 5 min in ice bath. It was then centrifuged at $16,000 \mathrm{~g}$ for $30 \mathrm{~min}$ at $4{ }^{\circ} \mathrm{C}$. The upper $75 \%$ of the centrifugation supernatant was taken and frozen to $-80^{\circ} \mathrm{C}$ and HFIP was removed using a vacuum freeze drier (Labconco, MO, USA). The pre-treated $\mathrm{A} \beta_{42}$ and $A \beta_{40}$ monomer sample was stored at $-20^{\circ} \mathrm{C}$. Prior to use, the $\mathrm{A} \beta_{42}$ and $\mathrm{A} \beta_{40}$ monomer sample was dissolved in $20 \mathrm{mM} \mathrm{NaOH}$ and sonicated for $5 \mathrm{~min}$ in ice bath. It was then diluted to a PBS solution ( $100 \mathrm{mM}$ phosphate buffer plus $10 \mathrm{mM} \mathrm{NaCl}, \mathrm{pH}$ 7.4) with or without an inhibitor. This solution was used for the following inhibition studies of $\mathrm{A} \beta$ aggregation and cytotoxicity.

ThT fluorescent assay. ThT is a widely used probe detecting amyloid fibrils or $A \beta$ aggregates ${ }^{45-47}$. A $\beta_{42}$ solutions $(25 \mu \mathrm{M}$ or $0.11 \mathrm{mg} / \mathrm{mL})$ prepared as described above were incubated with or without an inhibitor by continuous shaking at $150 \mathrm{rpm}$ in an air bath of $37^{\circ} \mathrm{C}$. Each condition of incubation was conducted in triplicate in three Eppendorf tubes and the average value is reported with standard deviation. The ThT FI was measured at different time intervals using fluorescence spectrometer of PE LS-55 (Perkin Elmer, MA, USA) at $25^{\circ} \mathrm{C}$ with a slit width of $5 \mathrm{~nm}$ and excitation and emission at 440 and $480 \mathrm{~nm}$, respectively. Before each measurement, $150 \mu \mathrm{L}$ samples were withdrawn at different time intervals and mixed uniformly with $3 \mathrm{~mL}$ ThT solutions $(25 \mu \mathrm{M}$ ThT in $100 \mathrm{mM}$ phosphate buffer, $\mathrm{pH}$ 7.4). The ThT FI of the samples without $\mathrm{A} \beta_{42}$ protein was subtracted as background from each read with $A \beta_{42}$ protein. Student's t-test (unpaired parametric test) was calculated for statistical comparisons to analyze the variance and $\mathrm{p}<0.05$ or less was considered to be statistically significant.

The kinetics of $A \beta_{40}$ aggregation was monitored by in situ ThT fluorescent assays in a microplate reader (TECAN Infinite, Salzburg, Austria). $\mathrm{A} \beta_{40}$ was adopted in the kinetic assays because it has a distinct lag phase for investigating the nucleation process. By contrast, $A \beta_{42}$ exhibits fast aggregation kinetics and in most cases no obvious lag phase could be observed ${ }^{48,49}$.

Observation of $\mathbf{A} \boldsymbol{\beta}_{42}$ aggregates. AFM is widely used to observe the morphologies of oligomeric structures, aggregates and mature amyloid fibrils ${ }^{50,51}$. The morphologies of $A \beta_{42}$ aggregates were observed by atomic force microscopy of model CSPM 5500 (Benyuan, Guangzhou, China). An AFM sample was prepared by dropping $10 \mu \mathrm{L}$ of $25 \mu \mathrm{M}$ pre-incubated $\mathrm{A} \beta_{42}$ sample on a piece of mica and allowing it air-dried before observation. The incubation conditions for the pre-incubation of $25 \mu \mathrm{M} \mathrm{A} \beta_{42}$ samples with or without inhibitors were the same as in the ThT assays.

Cell viability assay. The MTT method was employed for cell viability assay ${ }^{52,53}$ by using SH-SY5Y as a human neuronal cell model ${ }^{54,55}$. A total of $5 \times 10^{3}$ of SH-SY5Y cells $(80 \mu \mathrm{L})$ were cultured in DMEM/F12 medium containing $10 \% \mathrm{FBS}, 1 \%$ glutamic acid, $1 \%$ penicillin and streptomycin at $37^{\circ} \mathrm{C}$ under $5 \% \mathrm{CO}_{2}$ for $24 \mathrm{~h}$ in a 96-well plate. To assess the cytotoxicity induced by $\mathrm{A} \beta_{42}, 25 \mu \mathrm{M} \mathrm{A} \beta_{42}$ solutions $(20 \mu \mathrm{L})$ pre-incubated with or without an inhibitor for $16 \mathrm{~h}$ were added to the wells. The cells were incubated for another $48 \mathrm{~h}$, and then $10 \mu \mathrm{L}$ of $5.5 \mathrm{mg} / \mathrm{mL}$ MTT in the PBS solution was added into each well and incubated for another $4 \mathrm{~h}$. The suspension was centrifuged at $1,500 \mathrm{rpm}$ for $10 \mathrm{~min}$ to remove the supernatant. Then, $100 \mu \mathrm{L}$ DMSO was added to dissolve the formazan, followed by shaking at $150 \mathrm{rpm}$ for $10 \mathrm{~min}$. The cell viability was calculated using the absorbance value at $570 \mathrm{~nm}$ measured by a microplate reader (TECAN Infinite, Salzburg, Austria). The absorbance of the sample treated without cells was subtracted and the cell survival treated with the PBS only was set as control to normalize other data for comparison. Six replicates were conducted and the averaged data with standard deviations are reported. Student's t-test (unpaired parametric test) was calculated for statistical comparisons to analyze the variance and $\mathrm{p}<0.05$ or less was considered to be statistically significant.

Data availability. The datasets generated during and/or analyzed during the current study are available from the corresponding author on reasonable request.

\section{References}

1. Sikazwe, D., Yendapally, R., Ramsinghani, S. \& Khan, M. Alzheimer's drug discovery maze: a snap view of the past decades diverse pharmacological targets for the disorder. Mini-Rev. Med. Chem. 17, 305-318 (2017).

2. Xu, Z. et al. Age-dependent postoperative cognitive impairment and Alzheimer-related neuropathology in mice. Sci.Rep. 4, 3766 (2014).

3. Ashford, J. W. Treatment of Alzheimer's disease: the legacy of the cholinergic hypothesis, neuroplasticity, and future directions. J. Alzheimers. Dis. 47, 149-156 (2015).

4. Malmsten, M. et al. An ellipsometry-based Alzheimer plaque mimic: Effect of $\beta$-amyloid, lipoprotein identity and apolipoprotein E isoform. J. Colloid Interf. Sci. 276, 503-506 (2004).

5. Roy, S., Zhang, B., Lee, V. M. Y. \& Trojanowski, J. Q. Axonal transport defects: a common theme in neurodegenerative diseases. Acta neuropathol. 109, 5-13 (2005).

6. Hardy, J. \& Selkoe, D. J. The amyloid hypothesis of Alzheimer's disease: progress and problems on the road to therapeutics. Science 297, 353-356 (2002). 
7. Han, S. H., Park, J. C. \& Mook-Jung, I. Amyloid $\beta$-interacting partners in Alzheimer's disease: from accomplices to possible therapeutic targets. Prog. Neurobiol. 137, 17-38 (2016).

8. Bayer, T. A. \& Wirths, O. Focusing the amyloid cascade hypothesis on N-truncated abeta peptides as drug targets against Alzheimer's disease. Acta neuropathol. 127, 787-801 (2014).

9. Alam, P. et al. Vitamin k3 inhibits protein aggregation: Implication in the treatment of amyloid diseases. Sci. Rep. 6, 26759 (2016).

10. Noel, S., Cadet, S., Gras, E. \& Hureau, C. The benzazole scaffold: a SWAT to combat Alzheimer's disease. Chem. Soc. Rev. 42, 7747-7762 (2013).

11. Porat, Y., Abramowitz, A. \& Gazit, E. Inhibition of Amyloid Fibril Formation by Polyphenols: Structural Similarity and Aromatic Interactions as a Common Inhibition Mechanism. Chem. Biol. Drug. Des. 67, 27-37 (2006).

12. Goyal, D., Shuaib, S., Mann, S. \& Goyal, B. Rationally designed peptides and peptidomimetics as inhibitors of Amyloid- $\beta$ (A $\beta$ ) aggregation: potential therapeutics of Alzheimer's disease. ACS Comb. Sci. 19, 55-80 (2017).

13. Funke, S. A. \& Willbold, D. Peptides for Therapy and Diagnosis of Alzheimer's Disease. Curr. Pharm. Des. 18, 755-767 (2012).

14. Miles, L. A., Crespi, G. A. N., Doughty, L. \& Parker, M. W. Bapineuzumab captures the N-terminus of the Alzheimer's disease amyloid-beta peptide in a helical conformation. Sci. Rep. 3 (2013).

15. Huang, F. et al. Maintenance of amyloid $\beta$ peptide homeostasis by artificial chaperones based on mixed-shell polymeric micelles. Angew. Chem. Int. Edit. 53, 8985-8990 (2014).

16. Mirsadeghi, S. et al. Protein corona composition of gold nanoparticles/nanorods affects amyloid beta fibrillation process. Nanoscale 7, 5004-5013 (2015).

17. Kim, Y., Park, J. H., Lee, H. \& Nam, J. M. How Do the Size, Charge and Shape of Nanoparticles Affect Amyloid $\beta$ Aggregation on Brain Lipid Bilayer?. Sci. Rep. 6 (2016).

18. Chan, S. et al. Metal chelation, radical scavenging and inhibition of A $\beta 42$ fibrillation by food constituents in relation to Alzheimer's disease. Food Chem. 199, 185-194 (2016).

19. Kochi, A. et al. Inhibitory activity of curcumin derivatives towards metal-free and metal-induced amyloid- $\beta$ aggregation. Curr. Alzheimer Res. 12, 415-423 (2015).

20. Ono, K., Hasegawa, K., Naiki, H. \& Yamada, M. Curcumin has potent anti-amyloidogenic effects for Alzheimer's $\beta$-amyloid fibrils in vitro. J. Neurosci. Res. 75, 742-750 (2004).

21. Ahmad, E. et al. A mechanistic approach for islet amyloid polypeptide aggregation to develop anti-amyloidogenic agents for type-2 diabetes. Biochimie 93, 793-805 (2011).

22. Wu, C., Lei, H., Wang, Z., Zhang, W. \& Duan, Y. Phenol red interacts with the protofibril-like oligomers of an amyloidogenic hexapeptide NFGAIL through both hydrophobic and aromatic contacts. Biophys. J. 91, 3664-3672 (2006).

23. Thapa, A., Jett, S. D. \& Chi, E. Y. Curcumin attenuates amyloid- $\beta$ aggregate toxicity and modulates amyloid- $\beta$ aggregation pathway. ACS. Chem. Neurosci. 7, 56-68 (2015)

24. Palhano, F. L., Lee, J., Grimster, N. P. \& Kelly, J. W. Toward the molecular mechanism(s) by which EGCG treatment remodels mature amyloid fibrils. J. Am. Chem. Soc. 135, 7503-7510 (2013)

25. Ehrnhoefer, D. E. et al. EGCG redirects amyloidogenic polypeptides into unstructured, off-pathway oligomers. Nat. Struct. Mol. Biol. 15, 558-566 (2008)

26. Reinke, A. A. \& Gestwicki, J. E. Structure-activity relationships of amyloid beta-aggregation inhibitors based on curcumin: influence of linker length and flexibility. Chem. Biol. Drug Des. 70, 206-215 (2007).

27. Liu, F., Dong, X., He, L., Middelberg, A. P. \& Sun, Y. Molecular insight into conformational transition of amyloid $\beta$-peptide 42 inhibited by (-)-epigallocatechin-3-gallate probed by molecular simulations. J. Phys. Chem. B 115, 11879-11887 (2011).

28. Masuda, Y. et al. Solid-state NMR analysis of interaction sites of curcumin and 42-residue amyloid $\beta$-protein fibrils. Bioorgan. Med. Chem. 19, 5967-5974 (2011).

29. Yang, F. et al. Curcumin inhibits formation of amyloid $\beta$ oligomers and fibrils, binds plaques, and reduces amyloid in vivo. J. Biol. Chem. 280, 5892-5901 (2005).

30. Manju, S. \& Sreenivasan, K. Conjugation of curcumin onto hyaluronic acid enhances its aqueous solubility and stability. J. Colloid Interf. Sci. 359, 318-325 (2011).

31. Manju, S. \& Sreenivasan, K. Gold nanoparticles generated and stabilized by water soluble curcumin-polymer conjugate: blood compatibility evaluation and targeted drug delivery onto cancer cells. J. Colloid Interf. Sci. 368, 144-151 (2012).

32. Jiang, Z. et al. Multifunctionality of self-assembled nanogels of curcumin-hyaluronic acid conjugates on inhibiting amyloid $\beta$-protein fibrillation and cytotoxicity. React. Funct. Polym. 104, 22-29 (2016).

33. Aditya, N. P. et al. Co-delivery of hydrophobic curcumin and hydrophilic catechin by a water-in-oil-in-water double emulsion. Food Chem. 173, 7-13 (2015).

34. Zhu, S. et al. Lipase-catalyzed synthesis of acetylated EGCG and antioxidant properties of the acetylated derivatives. Food Res. Int. 56, 279-286 (2014).

35. Du, W. et al. Brazilin inhibits amyloid $\beta$-protein fibrillogenesis, remodels amyloid fibrils and reduces amyloid cytotoxicity. Sci. Rep. 5, 7992 (2015).

36. Shytle, R. D. et al. Optimized turmeric extracts have potent anti-amyloidogenic effects. Curr. Alzheimer Res. 6, 564-571 (2009).

37. Liu, H., Yu, L., Dong, X. \& Sun, Y. Synergistic effects of negatively charged hydrophobic nanoparticles and (-)-epigallocatechin-3gallate on inhibiting amyloid $\beta$-protein aggregation. J. Colloid Interf. Sci. 491, 305-312 (2017).

38. Cabaleiro-Lago, C., Szczepankiewicz, O. \& Linse, S. The effect of nanoparticles on amyloid aggregation depends on the protein stability and intrinsic aggregation rate. Langmuir 28, 1852-1857 (2012).

39. Arosio, P., Vendruscolo, M., Dobson, C. M. \& Knowles, T. P. Chemical kinetics for drug discovery to combat protein aggregation diseases. Trends Pharmacol. Sci. 35, 127-135 (2014).

40. Thal, D. R., Walter, J., Saido, T. C. \& Fandrich, M. Neuropathology and biochemistry of A $\beta$ and its aggregates in Alzheimer's disease. Acta Neuropathol. 129, 167-182 (2015).

41. Shoghi-Jadid, K. Imaging $\beta$-amyloid fibrils in Alzheimer's disease: a critical analysis through simulation of amyloid fibril polymerization. Nucl. Med. Biol. 32, 337-351 (2005).

42. Dahlgren, K. N. et al. Oligomeric and fibrillar species of amyloid- $\beta$ peptides differentially affect neuronal viability. J. Biol. Chem. 277, 32046-32053 (2002).

43. Xie, B., Li, X., Dong, X. \& Sun, Y. Insight into the inhibition effect of acidulated serum albumin on amyloid $\beta$-protein fibrillogenesis and cytotoxicity. Langmuir 30, 9789-9796 (2014).

44. Liang, K. et al. Targeted intracellular protein delivery based on hyaluronic acid-green tea catechin nanogels. Acta Biomater. 33, $142-152(2016)$

45. Groenning, M. Binding mode of thioflavin T and other molecular probes in the context of amyloid fibrils-current status. J. Chem. Biol. 3, 1-18 (2010)

46. Krebs, M. R., Bromley, E. H. \& Donald, A. M. The binding of thioflavin-T to amyloid fibrils: localisation and implications. J Struct. Biol. 149, 30-37 (2005).

47. Wolfe, L. S. et al. Protein-induced photophysical changes to the amyloid indicator dye thioflavin T. P. Natl. Acad. Sci. 107, 16863-16868 (2010).

48. Wu, C. et al. The structure of A 342 C-terminal fragments probed by a combined experimental and theoretical study. J. Mol. Biol. 387, 492-501 (2009). 
49. Jarrett, J. T., Berger, E. P. \& Lansbury, P. T. Jr The carboxy terminus of the $\beta$ amyloid protein is critical for the seeding of amyloid formation: Implications for the pathogenesis of Alzheimer's disease. Biochemistry 32, 4693-4697 (1993).

50. Tinker-Mill, C., Mayes, J., Allsop, D. \& Kolosov, O. V. Ultrasonic forcemicroscopy for nanomechanical characterization of early and late-stage amyloid-beta peptide aggregation. Sci. Rep. 4, 4004 (2014).

51. Lv, Z., Roychaudhuri, R., Condron, M. M., Teplow, D. B. \& Lyubchenko, Y. L. Mechanism of amyloid $\beta$-protein dimerization determined using single-molecule AFM force spectroscopy. Sci. Rep. 3 (2013).

52. Shearman, M. S. Toxicity of protein aggregates in PC12 cells: 3-(4,5-Dimethylthiazol-2-yl)-2,5-diphenyltetrazolium bromide assay. Methods Enzymol. 309, 716-723 (1999).

53. Fotakis, G. \& Timbrell, J. A. In vitro cytotoxicity assays: comparison of LDH, neutral Red, MTT and protein assay in Hepatoma cell lines following exposure to cadmium chloride. Toxicol. Lett. 160, 171-177 (2006).

54. Wang, Q. et al. Tanshinones inhibit amyloid aggregation by amyloid- $\beta$ peptide, disaggregate amyloid fibrils, and protect cultured cells. ACS Chem. Neurosci. 4, 1004-1015 (2013).

55. Thapa, A. et al. Biflavonoids are superior to monoflavonoids in inhibiting amyloid- $\beta$ toxicity and fibrillogenesis via accumulation of nontoxic oligomer-like structures. Biochemistry 50, 2445-2455 (2011).

\section{Acknowledgements}

This work was funded by the National Natural Science Foundation of China (Nos 91634119, 21376172 and 21621004) and the Natural Science Foundation of Tianjin from Tianjin Municipal Science and Technology Commission (Contract No. 16JCZDJC32300).

\section{Author Contributions}

X.D. and Y.S. designed the research; Z.J. and Y.X. performed the experiments. Y.L. helped the cytotoxicity assays. Z.J., X.D., L.Z. and Y.S. analyzed the data and wrote or contributed to the writing of the manuscript.

\section{Additional Information}

Supplementary information accompanies this paper at https://doi.org/10.1038/s41598-018-21933-6.

Competing Interests: The authors declare no competing interests.

Publisher's note: Springer Nature remains neutral with regard to jurisdictional claims in published maps and institutional affiliations.

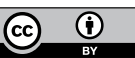

Open Access This article is licensed under a Creative Commons Attribution 4.0 International

License, which permits use, sharing, adaptation, distribution and reproduction in any medium or format, as long as you give appropriate credit to the original author(s) and the source, provide a link to the Creative Commons license, and indicate if changes were made. The images or other third party material in this article are included in the article's Creative Commons license, unless indicated otherwise in a credit line to the material. If material is not included in the article's Creative Commons license and your intended use is not permitted by statutory regulation or exceeds the permitted use, you will need to obtain permission directly from the copyright holder. To view a copy of this license, visit http://creativecommons.org/licenses/by/4.0/.

(C) The Author(s) 2018 